\title{
The Characteristic of Birefringence and Optical Loss in Femtosecond-Laser-Induced Region in terms of Nanogratings Distribution
}

\author{
Takafumi Ohfuchi* $^{* 1,2}$, Yuya Yamada*2, Masaaki Sakakura*1,3, Naoaki Fukuda*2, Toshio Takiya*2, Yasuhiko Shimotsuma*1 \\ and Kiyotaka Miura*1 \\ ${ }^{* 1}$ Department of Material Chemistry, Graduate School of Engineering, Kyoto University, Kyoto, \\ 615-8510, Japan \\ E-mail: ohfuchi@hitachizosen.co.jp \\ *2 Hitachi Zosen Corporation, Osaka, 551-0022, Japan \\ *3 Next Generation Laser Processing Technology Research Association, Kyoto 615-8245, Japan
}

\begin{abstract}
Femtosecond (fs) laser induced nanogratings formation can be applied to fabrication of complex birefringent optical elements, such as micro-arrayed waveplates, because birefringence appears in the region of nanogratings formation. For improvement of the quality of birefringent optics, it is important to find an optimized laser processing condition to obtain larger birefringence without loss of optical transmittance as possible. Therefore, it is important to elucidate the relation between birefringence, optical transmission loss and laser processing conditions. In this study, we have investigated the distribution of nanogratings in the modification by fs laser irradiation, retardance and transmittance under various processing conditions. We found that continuously distributed nanogratings with a homogeneous period in a modified region contribute to generation of birefringent region with larger retardance and higher transmittance.
\end{abstract}

DOI: $10.2961 /$ jlmn.2017.03.0008

Keywords: femtosecond laser, laser processing, nanogratings, birefringence, silica glass

\section{Introduction}

Femtosecond (fs) laser bulk processing inside a glass has been investigated extensively, because focused fs laser pulses inside a glass can induce local modifications, such as refractive index and highly etchable region, only in the focal volume without surface damages and birefringence appears in the modified region. Variety of functions can be added to various glasses by direct laser writing, such as optical waveguide, diffractive optical elements and microchannel [1, 2].

In some glasses, nanogratings are generated in the modified region after irradiation of multiple focused fs laser pulses [3-6]. One of the glasses is a silica glass, in which nanogratings formation was discovered for the first time and it has been investigated by a lot of researchers. Nanogratings inside a silica glass are due to the density of oxygen defects which contribute to periodically modulated refractive index [5]. Because of the periodic modulation of refractive index, the region of nanogratings presents birefringence. Because the orientation of nanogratings is determined by the direction of the linear polarization of fs laser pulses, the orientation of a birefringent structure can be controlled only by changing the polarization direction of the focused fs laser pulse. Therefore, complex optical elements, such as micro-arrayed waveplates, polarization imaging filter and 5-dimentioanl optical memory can be fabricated inside a silica glass easily by direct fs laser writing [7-9].

Among the applications, our target is a polarization imaging filter, which can analyze the polarization distribution and direction of detected light in a real-time. The thickness change of transparent materials and the stress distribution inside transparent materials can be obtained by polarization information [10]. A polarization imaging filter is made of periodically arrayed cells which are composed of birefringent cells of four different orientations [8]. To improve the quality of a polarization imaging filter, the retardance of the birefringent region should be near half of the wavelength of a detected light without the loss of optical transmittance. Therefore, it is necessary to elucidate laser processing conditions that gives larger birefringence with maintaining optical transmittance as possible.

A lot of researchers have investigated the relation between the retardance and nanogratings formation by fs laser processing inside a silica glass [11], and some of the researches have reported that the periodicity of nanogratings can be improved by an increasing pulse number [12-14]. However, nanogratings in their studies have been observed parallel to the laser propagation direction. To find the origins of the transmission loss and the retardance of birefringence in the laser modified region, the nanogratings distribution along the laser propagation direction should be observed, and the transmittance and retardance should be evaluated in terms of the nanogratings distribution along the laser propagation direction.

In this paper, we have investigated the nanogratings distribution along the laser propagation direction and the relation between the retardance and transmittance under various laser processing conditions. The retardance and transmittance of birefringent region were evaluated in terms of the nanogratings distribution along the laser propagation direction. 


\section{Experimental}

Figure 1 shows a direct fs laser writing system. Femtosecond laser (Cyber Laser Co, IFRIT) operating at $\lambda=780$ $\mathrm{nm}$ and a repetition rate of $1 \mathrm{kHz}$ was used in this system. The intensity distribution of the laser pulse was a top-hat distribution for using laser pulse energy effectively. The laser pulse was passed through a telescope (Magnification was 0.6) and focused inside a silica glass (VIOSIL-SQ, Shin-Etsu Quartz Products Co. Ltd.) by an objective lens (Nikon Corp, LU Plan FLUOR, $\times 20$, NA $=0.45$ ) at the depth of $50 \mu \mathrm{m}$ from the surface of a silica glass. The structural modifications were induced by nonlinear light absorption at the focus region. During the irradiation of fs laser pulses, the glass was translated perpendicular to the laser beam to write a straight line of birefringent structure and birefringent cell by controlling a linear-motor driven XYZ stage. The energies of focused laser pulses were controlled by an attenuator of a half-wave plate and polarizer before the telescope. The pulse duration was changed by a pulse compressor inside the laser source. The range of the pulse duration was between $220 \mathrm{fs}$ and $3000 \mathrm{fs}$. The polarization direction of the laser pulse was controlled by a half-wave plate in the telescope to be perpendicular to scanning direction.

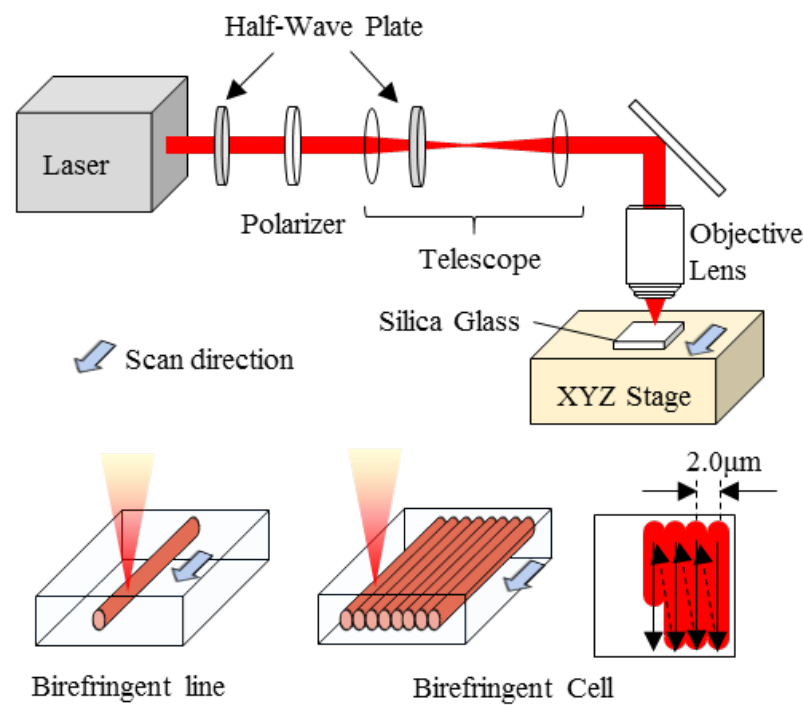

Fig. 1 Experimental setup for laser processing inside a silica glass by fs laser pulses.

The nanogratings distribution in the modified region was observed by an optical microscope and a Field Emission Scanning Electron Microscope (SEM; JEOL Ltd., JIB4600F) after polishing the cross-sections of laser written modification lines without deposition of the conductive coating.

The retardance of the birefringent line was measured by a polarization microscope with a liquid crystal (LC) compensator (CRI, Inc. LC-Polscope). A circularly polarized light at $546 \mathrm{~nm}$ was used, and the transmitted light was detected by a CCD after passing through an LC compensator. The distribution of the birefringence in the sample was obtained by analyzing four light intensity distributions measured at four different settings of the LC compensator.

The transmittance of a laser induced structure was evaluated by transmission optical microscope image of birefringent cell which was composed of multiple birefrin- gent lines arrayed with a space of $2 \mu \mathrm{m}$ inside a silica glass. The multiple birefringent lines were written by raster scanning, and the cell of $50 \mu \mathrm{m}^{2}$ was fabricated.

\section{Results and Discussion}

\subsection{Dependence on the number of irradiation pulses}

The irradiation pulse number per length was controlled by the scanning speed of a glass. We defined the irradiated pulse number per length considering the total pulse energy irradiated per unit area. For example, the pulse number was 100 pulses per $\mu \mathrm{m}$ at $10 \mu \mathrm{m} / \mathrm{s}$, because the repetition rate was $1 \mathrm{kHz}$. Figure 2 shows the dependence of retardance on the pulse number at the pulse energies of $1.0 \mu \mathrm{J}$ and 2.0 $\mu \mathrm{J}$ and pulse duration of $220 \mathrm{fs}$. The retardance increased as the pulse number increased and saturated at the pulse number of 100 pulses/ $\mu \mathrm{m}$ or more.

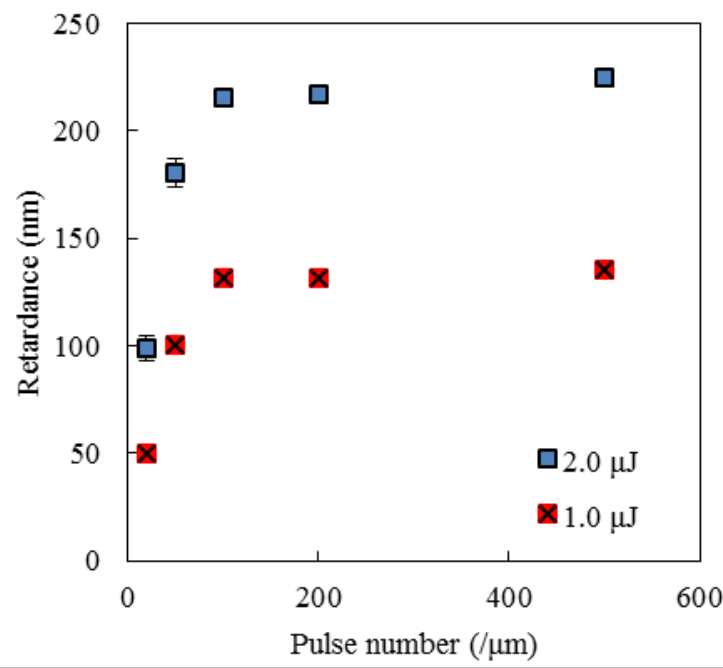

Fig. 2 Dependence of retardance on pulse number written at the pulse energies of $1.0 \mu \mathrm{J}$ and $2.0 \mu \mathrm{J}$.

Figure 3 shows the SEM images (upper images) and the optical microscope images (lower images) of cross-sections of the birefringent lines written from 20 pulses $/ \mu \mathrm{m}$ to 500 pulses $/ \mu \mathrm{m}$. While the length of laser modified regions along the laser axis were almost the same at any pulse number from the optical images in Fig. 3, the nanogratings distribution in the SEM images became discontinuous as the number of pulses decreased. Figure 4 is the magnified SEM images of Fig 3(a) and Fig. 3(d). The SEM image of the modification by less pulse number (50 pulses) show that nanogratings was fragmented (Fig. 4 (b)), while that by more pulse number shows that the nanogratings distribution became continuous over the laser modified region (Fig. 4 (a)). The fragmentation of nanogratings at fewer pulse number could result in smaller birefringence in the modified region, because the total number of nanogratings decreases due to the fragmentation. This expectation is supported by the pulse number dependence of the birefringence shown in Fig. 2, in which the retardance was decreased with decreasing the pulse number.

The nanogratings distribution in Fig. 4 (a) shows that the number of lines in nanogratings decreases at the lower region while the periodic of nanogratings was almost the same both in the top and bottom of laser modified region. This distribution suggests that the contribution from the 
lower part of the modified region to birefringence might be smaller.
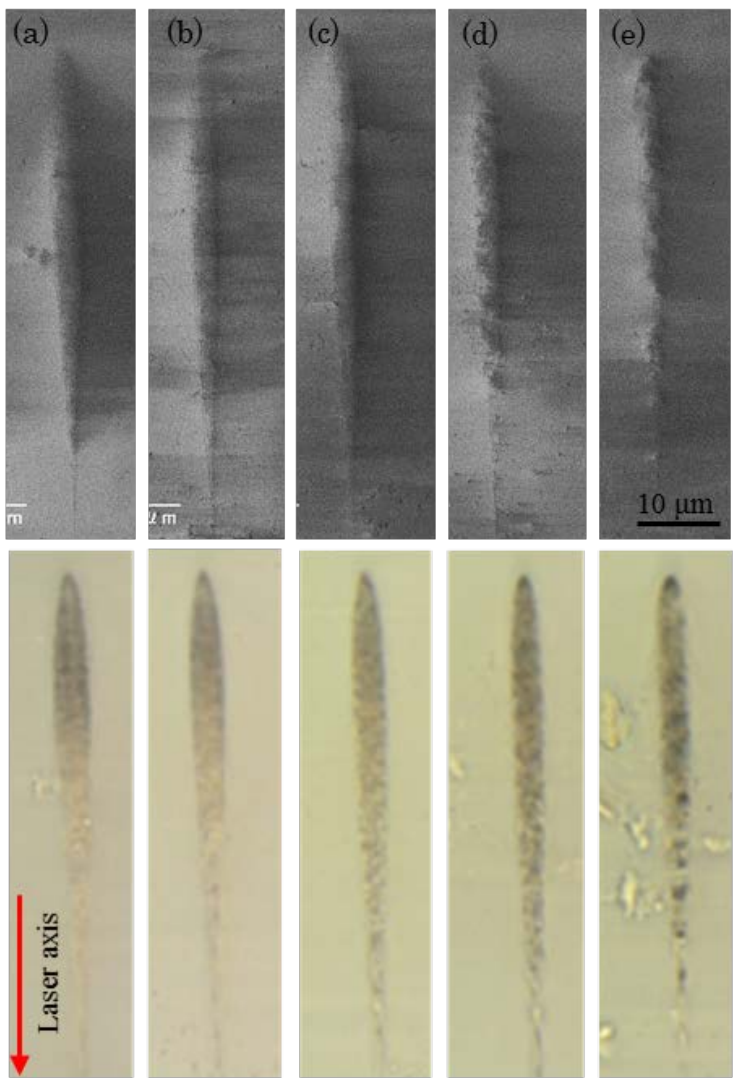

Fig. 3 SEM images (top) and optical microscope images (bottom) of laser induced structures written by (a) 500 pulses/ $\mu \mathrm{m}$, (b) 200 pulses $/ \mu \mathrm{m}$, (c) 100 pulses $/ \mu \mathrm{m}$, (d) $50 \mathrm{pulses} / \mu \mathrm{m}$, (e) $20 \mathrm{pulses} / \mu \mathrm{m}$.
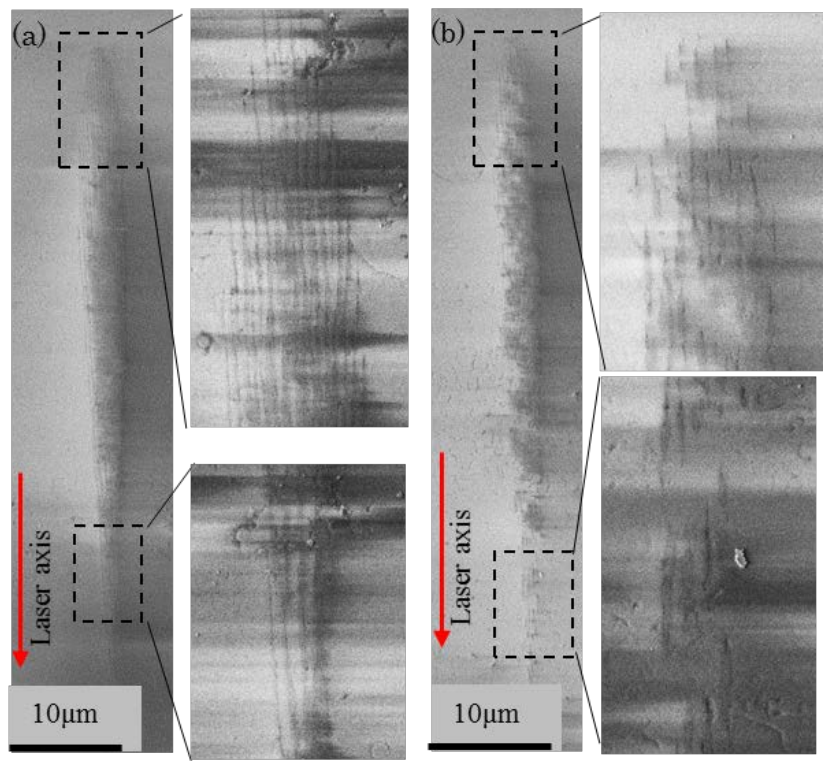

Fig.4 Magnified SEM images of cross-section of laser induced structures. (a) is magnified image of Fig. 3 (b) and (b) is magnified image of Fig. 3 (d).

Figure 5 shows birefringent cells of $50 \mu \mathrm{m}^{2}$ written at different scanning velocities, i.e. different pulse numbers per length. The transmittance of the laser induced region gradually increased with increasing the pulse number. The larger transmittance loss of laser induced region with fewer pulses could be attributed to the dark region in the crosssection observed in the optical microscope images of Fig. 3. The dark regions in the optical images in Fig. 3 indicate optical loss due to light scattering, and it was increased with decreasing the pulse number, especially in the lower region of the modification. Figure 6 shows optical microscope images of the side view of laser induced lines. We confirmed that refractive index distribution was smooth when the pulse number was 200 pulses $/ \mu \mathrm{m}$. On the other hand, refractive index distribution was inhomogeneous when the pulse number was 50 pulses/ $\mu \mathrm{m}$. From SEM images in Fig. 3, the part of fragmented nanogratings was increased with decreasing pulses. Therefore, the optical loss with smaller pulse number could come from the inhomogeneous distribution of refractive index due to the fragmented nanogratings. While the optical loss was along the scanning direction, the inhomogeneous distribution of refractive index could cause the optical loss along the laser axis due to Mie-scattering. In conclusion, the retardance and transmittance of the modified region became higher as the number of pulses increased.

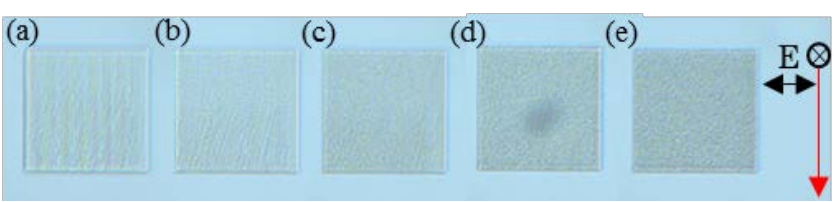

Fig. 5 Optical microscope images of laser induced birefringent cells of $50 \mu \mathrm{m}^{2}$ written by (a) 500 pulses $/ \mu \mathrm{m}$, (b) 200 pulses $/ \mu \mathrm{m}$, (c) $100 \mathrm{pulses} / \mu \mathrm{m}$, (d) $50 \mathrm{pulses} / \mu \mathrm{m}$, (e) 20 pulses $/ \mu \mathrm{m}$.
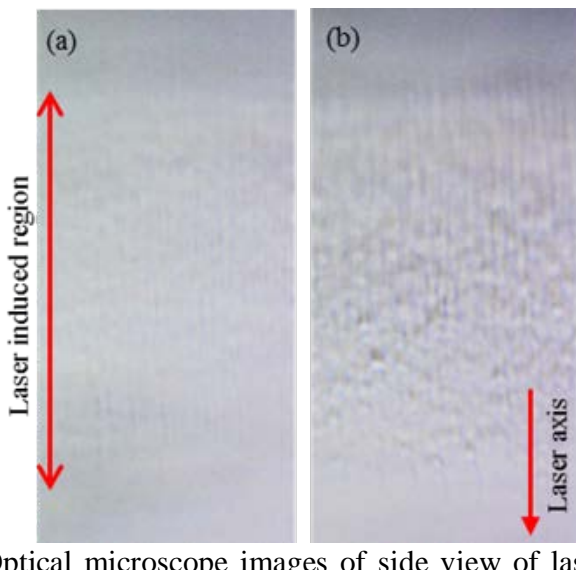

Fig. 6 Optical microscope images of side view of laser induced structures written by (a) 200 pulses $/ \mu \mathrm{m}$ (b) irradiated with 50 pulses $/ \mu \mathrm{m}$

\subsection{Dependence on the pulse duration}

The retardance of the laser induced birefringence region was measured as a function of pulse duration. Figure 7 shows the dependence of the retardance on the pulse duration at a pulse energy of $1.0 \mu \mathrm{J}$ and $2.0 \mu \mathrm{J}$, and a pulse number of 200 pulses/ $\mu \mathrm{m}$. The retardance increased slightly from 220 fs to $800 \mathrm{fs}$, but it decreased drastically at the pulse duration longer than $800 \mathrm{fs}$.

The SEM images and the optical microscope images of cross-sections of the birefringent lines are shown in Fig. 8. At pulse duration longer than $800 \mathrm{fs}$, the length of the laser induced structures were shorter and the regions of nanogratings were smaller and fragmented. Hence, drastic decrease of retardance over $800 \mathrm{fs}$ in Fig. 7 should be at- 
tributed to the shorter modified region and smaller region of nanogratings.

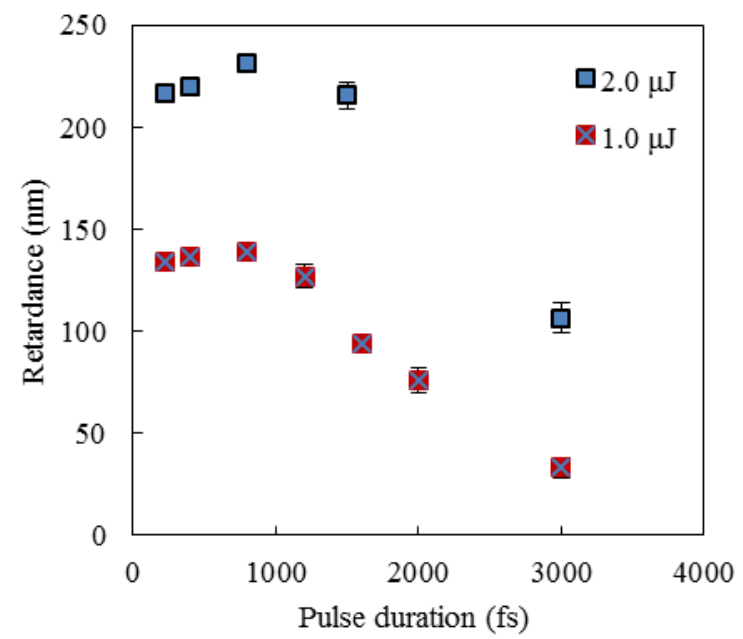

Fig. 7 Dependence of retardance and nanogratings length on pulse duration.

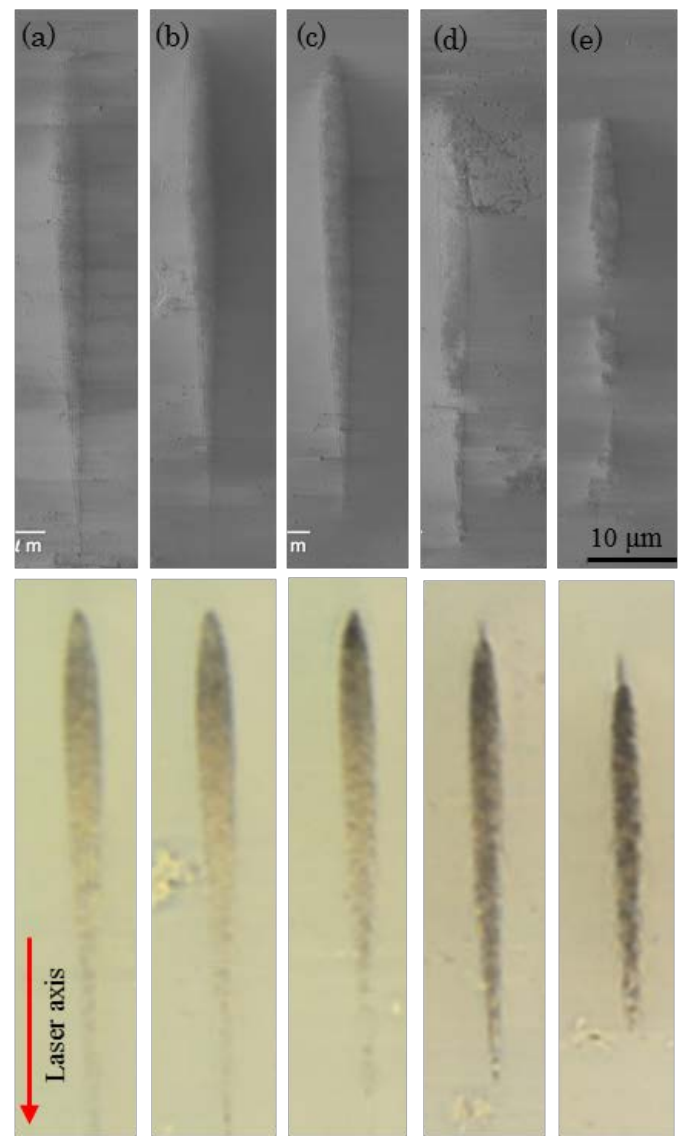

Fig. 8 SEM images (top) and optical microscope images (bottom) of laser induced structures written by (a) $220 \mathrm{fs}$, (b) $400 \mathrm{fs}$, (c) $800 \mathrm{fs}$, (d) $1600 \mathrm{fs}$, (e) $3000 \mathrm{fs}$.

Figure 9 shows birefringent cells of $50 \mu \mathrm{m}^{2}$ written at different pulse durations. The transmittance was lower at longer pulse duration, especially when the pulse duration was longer than 800 fs. By comparison of these images with the images of the cross-sections in Fig. 8, the loss of transmittance could be originated from the dark region of cross-sections in the optical microscope images. The nanogratings distribution along the laser axis became more continuous and the retardance and transmittance became higher at shorter pulse duration as well as the number of pulses increased. These results suggest that birefringent region of larger retardance and higher transmittance can be obtained by forming nanogratings distributed continuously over the laser modified region.

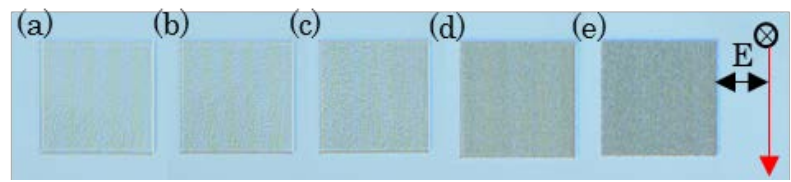

Fig. 9 Optical microscope images of laser induced birefringent cells of $50 \mu \mathrm{m}^{2}$ written by (a) $220 \mathrm{fs}$, (b) $400 \mathrm{fs}$, (c) $800 \mathrm{fs}$, (d) $1600 \mathrm{fs}$, (e) $3000 \mathrm{fs}$.

\subsection{Dependence on the pulse energy}

The retardance of the laser induced structure and the length of nanogratings in the laser modified regions along the laser axis were measured as functions of pulse energy. Birefringent lines were written by 220 fs laser pulses at 200 pulses/ $\mu \mathrm{m}$, because birefringent region with larger retardance and higher transmittance can be obtained by writing with short pulse duration and irradiation of many pulses. Figure 10 shows the dependence of the retardance of birefringent lines plotted against the pulse energy. The retardance increased with increasing pulse energy.

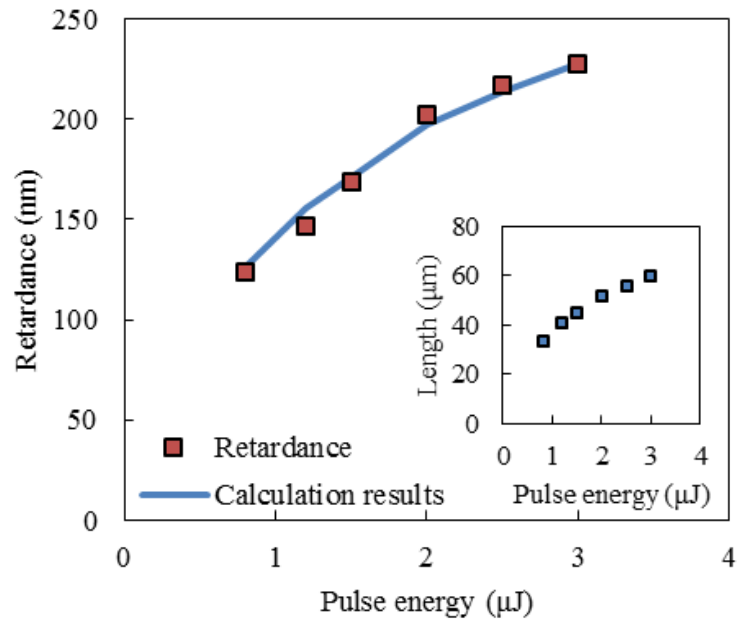

Fig. 10 Dependence of retardance on pulse energy.

The SEM images and the optical microscope images of cross-sections of the birefringent lines written at various pulse energies from $0.8 \mu \mathrm{J}$ to $2.0 \mu \mathrm{J}$ are shown in Fig. 11 . Nanogratings distributed continuously over the laser modified region at any pulse energy. The length of the nanogratings along the laser propagation directions were measured by the SEM images and plotted against the pulse energies in the inset of Fig. 10. The length of nanogratings increased with increasing pulse energy. This dependence on the pulse energy was almost the same as that of the retardance. The retardance in $\mathrm{nm}$ can be calculated by

$$
r=\Delta n \times l
$$

where $r$ is retardance, $\Delta n$ is birefringence and $l$ is length of birefringence. This equation means that the elongation of nanogratings structure at high energy could contribute to larger retardance. The blue line in Fig. 10 was calculated retardance with $\Delta n=0.0038$ and the length of nanogratings 
inset of Fig. 10, assuming that $\Delta n$ was constant over the laser induced structures. The retardance was strongly correlated with the length of nanogratings. The obtained value $\Delta n=0.0038$ is consistent with birefringence value of previous reports [15].
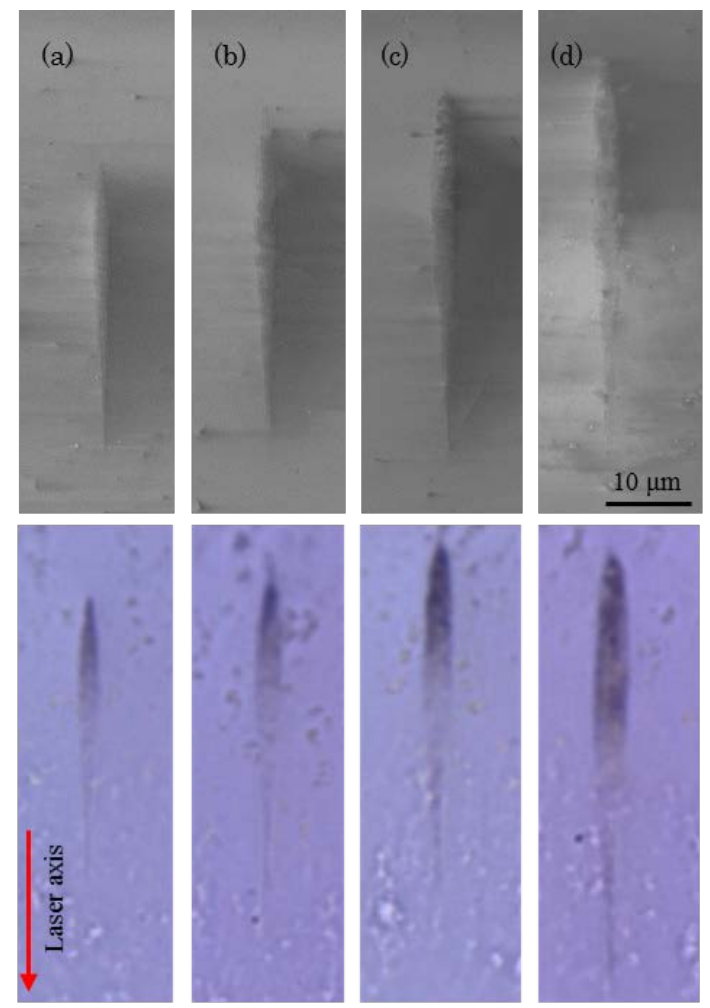

Fig. 11 SEM images (top) and optical microscope images (bottom) of laser induced structures written by (a) $0.8 \mu \mathrm{J}$, (b) $1.2 \mu \mathrm{J}$, (c) $1.5 \mu \mathrm{J}$, (d) $2.0 \mu \mathrm{J}$.

Figure 12 shows birefringent cells of $50 \mu \mathrm{m}^{2}$ written at different pulse energies. The transmittance of laser modified region became dark with increasing pulse energy. The dark region of cross-sections in the optical microscope images increased with increasing pulse energy as well as increasing pulse duration in Fig. 8, which could lead to optical loss. The retardance increased at large pulse energy, however, the dark region which could contribute to the loss of transmittance extended simultaneously.

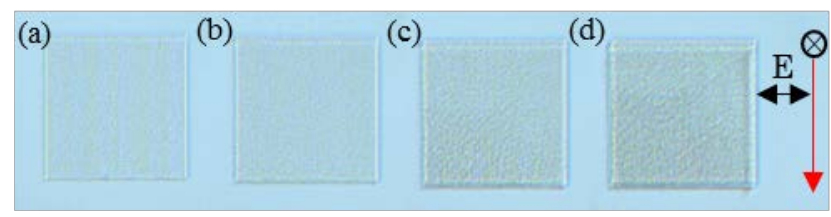

Fig. 12 Optical microscope images of laser induced birefringent cells of $50 \mu \mathrm{m}^{2}$ written by (a) $0.8 \mu \mathrm{J}$, (b) $1.0 \mu \mathrm{J}$, (c) $1.5 \mu \mathrm{J}$, (d) 2.0 $\mu \mathrm{J}$.

\section{Conclusion}

In this study, we investigated the retardance and transmittance in terms of nanogratings distribution of fs laser induced structures inside a silica glass at various pulse en- ergies, irradiated pulse number and pulse duration. As the result, we found that the birefringent region which is larger retardance and higher transmittance can be obtained at pulse duration of less than 800 fs and irradiated more than 100 pulses $/ \mu \mathrm{m}$. In addition, we found that the nanogratings distribution in the laser modified region became continuously and distributed over the laser modified region with the above conditions, and refractive index distribution is inhomogeneous in the fragmented nanogratings region which could cause the optical loss. Therefore, to from nanogratings distribution along the laser axis continuously is important for fabricating birefringent optics by fs laser writing.

\section{Acknowledgments and Appendixes}

This work was partially supported by Cross-Ministerial Strategic Innovation Promotion (SIP) Program.

\section{References}

[1] K. Miura, J. Qiu, H. Inouye, T. Mitsuyu and K. Hirao: Appl. Phys. Lett. 71, (1997) 3329.

[2] K. Sugioka and Y. Cheng: Appl. Phys. Rev. 1, (2014) 041303.

[3] Y. Shimotsuma, P. G. Kazansky, J. Qiu and K. Hirao: Phys. Rev. Lett. 91, (2003) 247405.

[4] Y. Shimotsuma, K. Hirao, J. Qiu and P. G. Kazansky: Mod. Phys. Lett. B 19, (2005) 225.

[5] R. Taylor, C. Hnatovsky and E. Simova: Laser \& Photon. Rev. 2, 1, (2008) 26.

[6] J. Canning, M. Lancry, K. Cook, A. Weickman, F. Brisset and B. Poumellec: Opt. Mater. Express 1, (2011) 998.

[7] Y. Shimotsuma, M. Sakakura, P. G. Kazansky, M. Beresna, J. Qiu, K. Miura and K. Hirao: Adv. Mater. 22, (2010) 4039.

[8] M. Beresna, M. Gecevicius and P. G. Kazansky: Opt. Mater. Express 1, (2011) 783.

[9] Y. Shimotsuma, K. Miura and K. Hirao: Int. J. Appl. Glass Sci. 4, (2013) 182.

[10] J. Vrijmoeth, R. W. Stok, R. Veldman, W. A. Schoonveld and T. M. Klapwijk: J. Appl. Phys. 83 (1998) 3816.

[11]L. Wang, Q. Chen, X. Cao, R. Buividas, X. Wang, S. Juodkazis and H. Sun: Light: Sci. Appl. 6, (2017) e17112.

[12] S. Richter, M. Heinrich, S. Doring, A. Tunnermann and S. Nolte: Appl. Phys. A 104, (2011) 503.

[13] S. Richter, M. Heinrich, S. Doring, A. Tunnermann, S. Nolte and U. Peschel: J. Laser Appl. 24, (2012) 042008.

[14] M. Beresna, M. Gecevicius, M. Lancry, B. Poumellec and P. G. Kazansky: Appl. Phys. Lett. 103 (2013) 131903.

[15]A. Murata, Y. Shimotsuma, M. Sakakura and K. Miura: J. Laser Micro/Nanoeng. 11, (2016) 95.

(Received: May 23, 2016, Accepted: October 17, 2017) 